\title{
Perturbations and transitivity for certain maps of an interval
}

\author{
MICHAE MISIUREWICZ \\ Institute of Mathematics, Warsaw University PKiN IX p.00-901, Warsaw, Poland
}

(Received 9 July 1982)

Abstract. We consider perturbations of certain transitive maps of an interval into itself and estimate how far from the transitivity the perturbed maps are. The distance turns out not to be of greater order than the square of the size of the perturbation.

\section{Introduction}

Denote by $\mathscr{C}_{s}$ the class of all maps $g:[0,1] \rightarrow[0,1]$ satisfying the following conditions:

$$
\begin{array}{cl}
g \text { is of class } C^{3} & \\
\qquad(0)=g(1)=0 & \text { for every } x \in[0,1] \\
g(1-x)=g(x) & \text { for every } x \in[0,1] \\
g^{\prime \prime}(x)<0 & \text { for every } x \in[0,1] \backslash\left\{\frac{1}{2}\right\}, \\
S g(x)<0 &
\end{array}
$$

where

$$
S g=g^{\prime \prime \prime} / g^{\prime}-\frac{3}{2}\left(g^{\prime \prime} / g^{\prime}\right)^{2}
$$

is the Schwarzian derivative of $g$. In fact, we do not need to use the third derivative of $g$, so we may replace $(0.1)$ and $(0.5)$ by

$$
\begin{gathered}
g \text { is of class } C^{2} \\
\left|g^{\prime}\right|^{-1 / 2} \text { is convex on }\left[0, \frac{1}{2}\right) \text { and }\left(\frac{1}{2}, 1\right]
\end{gathered}
$$

respectively (see [5], [2]). Notice that the condition $(0.3)$ may be viewed as the symmetry of $g$ with respect to $x=\frac{1}{2}$.

Consider a map $f \in \mathscr{C}_{s}$, satisfying additionally

$$
f\left(\frac{1}{2}\right)=1 \text {. }
$$

Such a map is topologically transitive (i.e. has a dense orbit) and has an invariant probabilistic measure, absolutely continuous with respect to the Lebesgue measure ([3], [5], [2]). Now we perturb $f$ slightly (but remain in $\mathscr{C}_{s}$ ) and we get a map $g \in \mathscr{C}_{s}$. It may happen that $g$ has quite different properties from $f$.

Then we may apply to $g$ a small random perturbation (of size $\delta>0$ ). Instead of moving from $x$ to $g(x)$, we move from $x$ to $g(x)+t$, where $t$ is chosen randomly from the interval $[-\delta, \delta]$. Here 'randomly' means randomly with respect to the 
Lebesgue measure, restricted to $[-\delta, \delta]$ and normalized (this is the simplest kind of a small random perturbation).

We want to answer the following question: how large should $\delta$ be (in comparison with the distance between $f$ and $g$ ) in order to restore the lost properties of $f$ ?

We cannot hope to make $g$ transitive on the whole $[0,1]$, unless

$$
\delta \geq \max \left(g^{2}\left(\frac{1}{2}\right), 1-g\left(\frac{1}{2}\right)\right) .
$$

This is due to the fact that the interval

$$
J_{g}=\left[g^{2}\left(\frac{1}{2}\right), g\left(\frac{1}{2}\right)\right]
$$

is $g$-invariant. But it is natural to restrict our attention to $J_{g}$.

Let us recall the notion of a pseudo-orbit. A g, $\delta$-pseudo-orbit from $x$ to $y$ is a sequence of points $\left(x_{0}, x_{1}, x_{2}, \ldots, x_{m}\right)$ such that

$$
x_{0}=x, \quad x_{m}=y \text { and }\left|x_{i+1}-g\left(x_{i}\right)\right|<\delta
$$

for $i=0,1, \ldots, m-1$. We shall say that the map $g$ is $\delta$-transitive if for every $x$, $y \in J_{g}$ there exists a $g, \delta$-pseudo-orbit from $x$ to $y$. The $\delta$-transitivity may be understood as the transitivity of a random perturbation of size $\delta$. It is known that if $g$ is $\delta$-transitive then there exists a probabilistic measure, absolutely continuous with respect to the Lebesgue measure, invariant for the process induced by our random perturbation, and with the support $\left[g^{2}\left(\frac{1}{2}\right)-\delta, g\left(\frac{1}{2}\right)+\delta\right]$.

Hence, we can modify our question to: how large should $\delta$ be (in comparison with the distance between $f$ and $g$ ) in order to make $g \delta$-transitive?

We shall assume that the distance between $f$ and $g$ is small in $C^{2}$-topology, but then we shall measure only the distance between $f\left(\frac{1}{2}\right)$ (i.e. 1$)$ and $g\left(\frac{1}{2}\right)$. We obtain the following result:

THEOREM A. For a given $f \in \mathscr{C}_{s}$ with $f\left(\frac{1}{2}\right)=1$, there exists a constant $c>0$ such that if $g$ is sufficiently close to $f$ in $C^{2}$-topology, then $g$ is $c\left(1-g\left(\frac{1}{2}\right)\right)^{2}$-transitive.

Since we also want to be able to obtain some numerical estimates for a given $g$, we shall prove the following theorem:

THEOREM B. There exist positive constants $c_{8}$ and $c_{9}$, depending continuously on $g$ in $C^{2}$-topology, such that if $1-g\left(\frac{1}{2}\right)<c_{8}$ then $g$ is $c_{9} \cdot\left(1-g\left(\frac{1}{2}\right)\right)^{2}$-transitive.

Since for the map $f$,

$$
1-f\left(\frac{1}{2}\right)=0<c_{8}(f),
$$

theorem A follows immediately from theorem $B$.

The constants $c_{8}$ and $c_{9}$ can be calculated for a given $g$. We shall do it for maps of a form $x \mapsto r x(1-x)$.

\section{Constants}

We have to define several constants. Perhaps it would be more convenient to the reader to have them all defined in one section.

The quantity that appears in both theorems is

$$
\eta=1-g\left(\frac{1}{2}\right) \text {. }
$$


As we shall see later, our assumptions will imply that $g$ has two fixed points, both of them repelling (lemma 1). One of them is 0 ; call the other one $a^{\prime}$. Thus,

$$
g\left(a^{\prime}\right)=a^{\prime}, \quad a^{\prime} \neq 0 .
$$

Now we can define

$$
\begin{gathered}
a=1-a^{\prime} \\
c_{1}=g^{\prime}(a)\left(g^{\prime}(a)-1\right)\left[g^{\prime}(0)\left(g^{\prime}(0)-1\right)\right]^{-1} .
\end{gathered}
$$

Since both fixed points are repelling, we have

$$
g^{\prime}(0)-1>0 \text { and } g^{\prime}(a)-1=\left|g^{\prime}\left(a^{\prime}\right)\right|-1>0,
$$

and consequently, $c_{1}>0$.

Since $g^{\prime \prime}$ is strictly negative and bounded, there exist positive constants $c_{2}$ and $c_{3}$ such that

$$
2 c_{2} t \leq\left|g^{\prime}\left(\frac{1}{2}+t\right)\right| \leq 2 c_{3} t \text { for all } t \in\left[-\frac{1}{2}, \frac{1}{2}\right]
$$

(to be sure that $c_{2}$ and $c_{3}$ depend continuously on $g$, we can take

$$
\left.c_{2}=\frac{1}{2} \inf _{[0,1]}\left|g^{\prime \prime}\right|, \quad c_{3}=\frac{1}{2} \sup _{[0,1]}\left|g^{\prime \prime}\right|\right) \text {. }
$$

We define further:

$$
\begin{aligned}
& c_{4}=2 a c_{1} c_{2} \\
& c_{5}=\left(g^{\prime}(0)-1\right) c_{2}^{-1} \\
& c_{6}=\left(c_{5} \eta\right)^{\frac{1}{2}} \\
& c_{7}=6 c_{4}^{-1} \eta \\
& c_{8}=\min \left(\frac{1}{16} c_{3}^{-2} c_{4}^{2} c_{5}^{-1} \ln ^{2}\left(c_{3} c_{5}+1\right), \frac{1}{54} c_{3}^{-1} c_{4}^{2}, a \cdot\left(g^{\prime}(0)\right)^{-1}, \frac{1}{2}\right) \\
& c_{9}=36 c_{3} c_{4}^{2} .
\end{aligned}
$$

In the following, when we say 'if $\eta<c_{8} \cdots$ ', we mean: 'if $\eta<\frac{1}{2}$, (then $a$ exists) 'and $\eta<a \cdot\left(g^{\prime}(0)\right)^{-1}$, (then the constants $c_{1}, \ldots$ are positive) 'and $\eta<\min \left(\frac{1}{16} c_{3}^{-2} c_{4}^{2} c_{5}^{-1} \ln ^{2}\left(c_{3} c_{5}+1\right), \frac{1}{5_{4}} c_{3}^{-1} c_{4}^{2}\right) \ldots$.

One can see that although formally there are no constants depending continuously on $g$ for all $g \in \mathscr{C}_{s}$, this is enough to deduce theorem A from theorem B. However, we can make a minor change and define instead of $c_{8}$ the constant $c_{8}^{\prime}$ :

If

$$
\eta \geq \frac{1}{2}
$$

or

$$
a \cdot\left(g^{\prime}(0)\right)^{-1} \leq \eta<\frac{1}{2}
$$

or

$$
\min \left(\frac{1}{16} c_{3}^{-2} c_{4}^{2} c_{5}^{-1} \ln ^{2}\left(c_{3} c_{5}+1\right), \frac{1}{54} c_{3}^{-1} c_{4}^{2}\right) \leq \eta<\min \left(\frac{1}{2}, a \cdot\left(g^{\prime}(0)\right)^{-1}\right),
$$

then $c_{8}^{\prime}=\eta$;

if

$$
\eta<\frac{1}{2} \text { and } \eta<a \cdot\left(g^{\prime}(0)\right)^{-1}
$$


and

$$
\eta<\min \left(\frac{1}{16} c_{3}^{-2} c_{4}^{2} c_{5}^{-1} \ln ^{2}\left(c_{3} c_{5}+1\right), \frac{1}{54} c_{3}^{-1} c_{4}^{2}\right)
$$

then $c_{8}^{\prime}=c_{8}$.

Then $c_{8}^{\prime}$ will be defined and continuous for all $g \in \mathscr{C}_{s}$, and $\eta<c_{8}$ if and only if $\eta<c_{8}^{\prime}$.

Notice, that if $\eta<\frac{1}{2}$ and $\eta<a \cdot\left(g^{\prime}(0)\right)^{-1}$, then

$$
\frac{1}{54} c_{3}^{-1} \cdot c_{4}^{2}<\min \left(\frac{1}{2}, a \cdot\left(g^{\prime}(0)\right)^{-1}\right) .
$$

We obtain this because

$$
c_{1}<1, \quad a<\frac{1}{2}, \quad g^{\prime}(0) \leq c_{3}, \quad c_{2} \leq c_{3} \quad \text { and } \quad c_{2} \leq 4 .
$$

(This holds since

$$
\left.1 \geq 1-\eta \geq \int_{0}^{1} \int_{\frac{1}{2}}^{\frac{1}{2}+x} 2 c_{2} d t d x=2 c_{2} \cdot \frac{1}{8} .\right)
$$

We have:

and

$$
\begin{aligned}
\frac{1}{54} c_{3}^{-1} c_{4}^{2} & =\frac{2}{27} a^{2} c_{1}^{2} c_{2}^{2} c_{3}^{-1} \\
& \leq \frac{2}{27} \cdot \frac{1}{4} \cdot 1 \cdot 4 \cdot 1 \\
& =\frac{2}{27}<\frac{1}{2}
\end{aligned}
$$

$$
\begin{aligned}
\frac{1}{54} c_{3}^{-1} c_{4}^{2} & =\frac{2}{27} a^{2} c_{1}^{2} c_{2}^{2} c_{3}^{-1} \\
& =\frac{2}{27} \cdot a \cdot \frac{1}{2} \cdot 1 \cdot 16 \cdot\left(g^{\prime}(0)\right)^{-1} \\
& =\frac{16}{27} a \cdot\left(g^{\prime}(0)\right)^{-1}<a \cdot\left(g^{\prime}(0)\right)^{-1} .
\end{aligned}
$$

\section{Proof of theorem $B$}

We have to analyse the structure of the map $g$. We shall proceed through a sequence of lemmas.

We start with the proof of the fact already used in the previous section:

LEMMA 1. If $\eta<\frac{1}{2}$ then $g$ has exactly 2 fixed points. If additionally $\eta<a \cdot\left(g^{\prime}(0)\right)^{-1}$ then they are both repelling and the topological entropy of $g$ is at least $\frac{1}{2} \log 2$.

Proof. If $\eta<\frac{1}{2}$ then $g\left(\frac{1}{2}\right)-\frac{1}{2}>0$, and consequently there is exactly one fixed point, $a^{\prime}$, on $\left[\frac{1}{2}, 1\right]$. Since $g^{\prime \prime}<0$, for any $x \in\left(0, \frac{1}{2}\right)$ we have

$$
g(x)>2 x \cdot g\left(\frac{1}{2}\right)+(1-2 x) \cdot g(0)>x,
$$

and consequently, 0 is the only fixed point of $g$ on $\left[0, \frac{1}{2}\right]$.

If additionally $\eta<a \cdot\left(g^{\prime}(0)\right)^{-1}$, we have:

$$
g^{2}\left(\frac{1}{2}\right)=g(\eta) \leq g^{\prime}(0) \cdot \eta<a
$$

and

$$
g^{3}\left(\frac{1}{2}\right)<g(a)=a^{\prime},
$$

and therefore:

$$
\begin{aligned}
& g\left(\left[a^{\prime}, g\left(\frac{1}{2}\right)\right]\right) \supset\left[g^{2}\left(\frac{1}{2}\right), \frac{1}{2}\right] \cup\left[\frac{1}{2}, a^{\prime}\right], \\
& g\left(\left[g^{2}\left(\frac{1}{2}\right), \frac{1}{2}\right]\right) \supset\left[a^{\prime}, g\left(\frac{1}{2}\right)\right], \\
& g\left(\left[\frac{1}{2}, a^{\prime}\right]\right) \supset\left[a^{\prime}, g\left(\frac{1}{2}\right)\right] .
\end{aligned}
$$


Hence,

$$
h(g) \geq \frac{1}{2} \log 2
$$

(cf. [1]).

We have

$$
g^{\prime}(0)>g\left(\frac{1}{2}\right) \cdot\left(\frac{1}{2}\right)^{-1}>1 .
$$

Since $S g>0$, if $\left|g^{\prime}\left(a^{\prime}\right)\right| \leq 1$ then $h(g)=0$ (see e.g. [2]) which is a contradiction. Hence also $\left|g^{\prime}\left(a^{\prime}\right)\right|>1$.

It is known ([4], [2]) that a map $g \in \mathscr{C}_{s}$ is semi-conjugate to a piecewise linear map $\tilde{\mathrm{g}}:[0,1] \rightarrow[0,1]$ with constant slope and the same entropy. We shall denote this semi-conjugacy by $p$. For $x \in[0,1]$, we set

$$
P(x)=p^{-1}(\{p(x)\}) .
$$

The map $p$ is non-decreasing, and hence $P(x)$ is either a proper interval or consists of one point. Set $K=P\left(\frac{1}{2}\right)$.

We shall analyse closer the case when $K$ is a proper interval. It is known ([3], [2]) that $\frac{1}{2}$ is then periodic for $\tilde{\boldsymbol{g}}$. We shall denote by $n$ its prime period. It can be easily seen that $K$ is symmetric with respect to $\frac{1}{2}$. Hence, we have the following situation:

$$
\begin{gathered}
K=\left[\frac{1}{2}-b, \frac{1}{2}+b\right], \\
g^{n}(K) \subset K .
\end{gathered}
$$

Since we have to be able to 'jump out' from the orbit of $K$ by a $g, \delta$-pseudo-orbit, our main goal will be to estimate $b$.

We begin by taking a point

$$
x_{\varepsilon}=\frac{1}{2}+\varepsilon \in\left(\frac{1}{2}, a^{\prime}\right)
$$

and looking at its trajectory and the derivatives along it. We have $g\left(x_{\varepsilon}\right)>a^{\prime}$. Then the trajectory stays for some time (this time may be 0$)$ at $(0, a]$ and then for some $j>1$ we have $g^{j}\left(x_{\varepsilon}\right)>a$. We take the smallest $j$ with this property.

LEMMA 2. We have

$$
\left|\left(g^{j-1}\right)^{\prime}\left(g\left(x_{\varepsilon}\right)\right)\right|>a c_{1}\left(1-g\left(x_{\varepsilon}\right)\right)^{-1} .
$$

Proof. Instead of looking at $g\left(x_{\varepsilon}\right)$, we look at

$$
y_{0}=1-g\left(x_{\varepsilon}\right) \text {. }
$$

Set $y_{k}=g^{k}\left(y_{0}\right), k=1,2, \ldots, j-1$. Clearly,

$$
y_{k}=g^{k+1}\left(x_{\varepsilon}\right) \text {, }
$$

and hence $y_{0}, y_{1}, \ldots, y_{j-2} \leq a$, whereas $y_{j-1}>a$. The derivative of $g$ is decreasing on $\left[0, \frac{1}{2}\right]$, and therefore

$$
y_{j-1}-y_{j-2} \leq\left(y_{1}-y_{0}\right) \cdot\left(g^{i-2}\right)^{\prime}\left(y_{0}\right) .
$$

But

$$
g^{\prime}(a)<\frac{y_{j-1}}{y_{i-2}}<g^{\prime}(0) \quad \text { and } \quad \frac{y_{1}}{y_{0}}<g^{\prime}(0) \text {, }
$$


and hence

$$
\left(g^{j-2}\right)^{\prime}\left(y_{0}\right) \geq \frac{y_{j-1}-y_{j-2}}{y_{1}-y_{0}}>\frac{\left(g^{\prime}(a)-1\right) y_{j-2}}{\left(g^{\prime}(0)-1\right) y_{0}}>\frac{\left(g^{\prime}(a)-1\right) y_{j-2}}{\left(g^{\prime}(0)-1\right) y_{0} \cdot g^{\prime}(0)}>\frac{a}{y_{0}} \frac{g^{\prime}(a)-1}{\left(g^{\prime}(0)-1\right) g^{\prime}(0)} .
$$

Since

$$
g^{\prime}\left(y_{j-2}\right) \geq g^{\prime}(a)
$$

we get

$$
\left(g^{j-1}\right)^{\prime}\left(y_{0}\right)>\frac{a}{y_{0}} \cdot c_{1} .
$$

LEMMA 3. We have

$$
c_{2} \varepsilon^{2} \leq 1-g\left(x_{\varepsilon}\right)-\eta \leq c_{3} \varepsilon^{2} .
$$

Proof. From (1.5) we get

$$
\begin{aligned}
c_{2} \cdot \varepsilon^{2} & =\int_{0}^{\varepsilon} 2 c_{2} t d t \leq \int_{0}^{\varepsilon}\left|g^{\prime}\left(\frac{1}{2}+t\right)\right| d t \\
& \leq \int_{0}^{\varepsilon} 2 c_{3} t d t=c_{3} \varepsilon^{2}
\end{aligned}
$$

but

$$
\int_{0}^{\varepsilon}\left|g^{\prime}\left(\frac{1}{2}+t\right)\right| d t=\left|g\left(x_{\varepsilon}\right)-g\left(\frac{1}{2}\right)\right|=1-\eta-g\left(x_{\varepsilon}\right)
$$

LEMMA 4. We have

$$
\left|\left(g^{j}\right)^{\prime}\left(x_{\varepsilon}\right)\right|>c_{4} \varepsilon\left(\eta+c_{3} \varepsilon^{2}\right)^{-1}
$$

Proof. Since

$$
\left|\left(g^{j}\right)^{\prime}\left(x_{\varepsilon}\right)\right|=\left|g^{\prime}\left(x_{\varepsilon}\right)\right| \cdot\left|\left(g^{j-1}\right)^{\prime}\left(g\left(x_{\varepsilon}\right)\right)\right|,
$$

we get from lemma 2 and (1.5),

$$
\left|\left(g^{j}\right)^{\prime}\left(x_{\varepsilon}\right)\right|>2 c_{2} \varepsilon \cdot a c_{1}\left(1-g\left(x_{\varepsilon}\right)\right)^{-1} .
$$

By lemma 3 we have

$$
1-g\left(x_{\varepsilon}\right)=\left(1-\eta-g\left(x_{\varepsilon}\right)\right)+\eta \leq c_{3} \varepsilon^{2}+\eta,
$$

and hence

$$
\left|\left(g^{j}\right)^{\prime}\left(x_{\varepsilon}\right)\right|>c_{4} \varepsilon\left(\eta+c_{3} \varepsilon^{2}\right)^{-1}
$$

Take a point $x \in[0,1]$. If the orbit of $x$ comes closer to $\frac{1}{2}$ then $x$ itself (or if $x$ is periodic), we may denote

$$
k(x)=\min \left\{i \geq 1:\left|g^{i}(x)-\frac{1}{2}\right| \leq\left|x-\frac{1}{2}\right|\right\} .
$$


Analysing carefully the proof from [3], we get the following lemma:

LEMMA 5. If $x \notin K$ then

$$
\left|\left(g^{k(x)}\right)^{\prime}(x)\right|>1 .
$$

By using lemma 4 once and then perhaps lemma 5 several times, we obtain

LEMMA 6. For all $\varepsilon \in[0, b]$,

$$
\left|\left(g^{n}\right)^{\prime}\left(x_{\varepsilon}\right)\right|>c_{4} \varepsilon\left(\eta+c_{3} \varepsilon^{2}\right)^{-1} .
$$

Define a function $F$ by:

$$
F(t)=2 t-\frac{1}{2} c_{3}^{-1} c_{4} \ln \left(\eta^{-1} c_{3} t^{2}+1\right) .
$$

LEMMA 7. We have $F(b)>0$.

Proof. Since $g^{n}$ maps $\left[\frac{1}{2}, b\right]$ homeomorphically onto some sub-interval of $K$, we have

$$
\begin{aligned}
2 b & \geq \int_{0}^{b}\left|\left(g^{n}\right)^{\prime}\left(x_{\varepsilon}\right)\right| d \varepsilon>\int_{0}^{b} c_{4} \varepsilon\left(\eta+c_{3} \varepsilon^{2}\right)^{-1} d \varepsilon \\
& =c_{4} c_{3}^{-1} \int_{0}^{b} \varepsilon \cdot\left(\eta c_{3}^{-1}+\varepsilon^{2}\right)^{-1} d \varepsilon \\
& =c_{3}^{-1} c_{4}\left[\frac{1}{2} \ln \left(\eta c_{3}^{-1}+\varepsilon^{2}\right)\right]_{\varepsilon=0}^{\varepsilon=b} \\
& =\frac{1}{2} c_{3}^{-1} c_{4} \ln \left[\left(\eta c_{3}^{-1}+b^{2}\right) \cdot\left(c_{3}^{-1} \eta\right)^{-1}\right] \\
& =\frac{1}{2} c_{3}^{-1} c_{4} \ln \left(\eta^{-1} c_{3} b^{2}+1\right) .
\end{aligned}
$$

We are going to obtain an estimation of $b$ from lemma 6 . However, we shall first need a coarser estimate, derived in a different way.

LEMMA 8. We have $b<c_{6}$.

Proof. Set $d=1-g\left(x_{b}\right)$. Then, by lemma 3,

$$
d-\eta \geq c_{2} b^{2} .
$$

Suppose that $g^{\prime}(0) \cdot \eta \leq d$. Then $g(\eta)<d$, and an interval symmetric to $g(K)$ with respect to $\frac{1}{2}$, contains a fundamental domain of $g$ near 0 . Therefore, the union of its images contains whole $J_{g}$. This is impossible under our assumptions. Hence, $g^{\prime}(0) \eta>d$, and consequently

$$
\left(g^{\prime}(0)-1\right) \eta>d-\eta \geq c_{2} b^{2} .
$$

By (1.7) and (1.8), we get $b<c_{6}$.

Lemma 9. Assume that $F\left(c_{6}\right)<0$. Then there exists exactly one $t_{0} \in\left(0, c_{6}\right)$ such that $F\left(t_{0}\right)=0$.

Proof. We have

$$
F^{\prime}(t)=2-c_{4} t\left(\eta+c_{3} t^{2}\right)^{-1} .
$$

Since $F(0)=0, F^{\prime}(0)=2>0$ and $F\left(c_{6}\right)<0$, such a $t_{0}$ exists. Suppose that it is not unique. Then the equation $F^{\prime}(0)=0$ has at least 3 roots on $\left(0, c_{6}\right)$. But this equation is equivalent to a quadratic equation - a contradiction. 
Lemma 10. Assume that

$$
c_{3} c_{7}^{2} \eta^{-1} \leq \frac{2}{3}
$$

Then $F\left(c_{7}\right) \leq 0$.

Proof. We have

$$
\ln (t+1) \geq t-\frac{1}{2} t^{2} \quad \text { for all } t>-1
$$

and hence

$$
\begin{aligned}
F\left(c_{7}\right) & \leq 2 c_{7}-\frac{1}{2} c_{3}^{-1} c_{4}\left[\eta^{-1} c_{3} c_{7}^{2}-\frac{1}{2}\left(\eta^{-1} c_{3} c_{7}^{2}\right)^{2}\right] \\
& \leq 2 c_{7}-\frac{1}{2} c_{3}^{-1} c_{4}\left(\eta^{-1} c_{3} c_{7}^{2}-\frac{1}{2} \eta^{-1} c_{3} c_{7}^{2} \cdot \frac{2}{3}\right) \\
& =2 c_{7}-\frac{1}{2} c_{3}^{-1} c_{4} \frac{2}{3} \eta^{-1} c_{3} c_{7}^{2} \\
& =c_{7}\left(2-\frac{1}{3} c_{4} \eta^{-1} c_{7}\right)=0 .
\end{aligned}
$$

Lemma 11. Assume that:

(i) $F\left(c_{6}\right)<0$,

(ii) $c_{3} c_{7}^{2} \eta^{-1} \leq \frac{2}{3}$.

Then $b<c_{7}$.

Proof. Suppose that $b \geq c_{7}$. By lemma 8, we then have $c_{7} \leq b<c_{6}$. But then:

(a) by (ii) and lemma $10, \quad F\left(c_{7}\right) \leq 0$;

(b) by lemma 7, $\quad F(b)>0$;

(c) by (i), $\quad F\left(c_{6}\right)<0$;

and consequently the equation $F(t)=0$ has at least two roots on the interval $\left[c_{7}, c_{6}\right)$. But, in view of (i), this contradicts lemma 9.

We can find on the trajectory of $K$ an interval shorter than $K$ itself.

LEMMA 12. If $\eta<c_{8}$ then the length of $g(K)$ is smaller than $c_{9} \cdot \eta^{2}$.

Proof. Assume that $\eta<c_{8}$. Since

$$
c_{8} \leq \frac{1}{16} c_{3}^{-2} c_{4}^{2} c_{5}^{-1} \ln ^{2}\left(c_{3} c_{5}+1\right)
$$

we have

$$
16 c_{5} \eta<c_{4}^{2} c_{3}^{-2} \ln ^{2}\left(c_{3} c_{5}+1\right)
$$

Since $c_{6}^{2}=c_{5} \eta$, we get

$$
4 c_{6}<c_{4} c_{3}^{-1} \ln \left(c_{3} c_{6}^{2} \eta+1\right)
$$

and hence

$$
2 c_{6}<\frac{1}{2} c_{3}^{-1} c_{4} \ln \left(\eta^{-1} c_{3} c_{6}+1\right)
$$

i.e. $F\left(c_{6}\right)<0$.

Since

$$
c_{8} \leq \frac{1}{54} c_{3}^{-1} c_{4}^{2}
$$

we have

$$
36 \eta c_{3} c_{4}^{-2} \leq \frac{2}{3}
$$


i.e.

$$
c_{3} c_{7}^{2} \eta^{-1} \leq \frac{2}{3}
$$

Thus, the assumptions of lemma 11 are satisfied. Therefore, $b<c_{7}$. By lemma 3 we obtain that the length of $g(K)$ is not larger than

$$
c_{3} b^{2}<c_{3} c_{7}^{2}=36 c_{3} c_{4}^{-2} \eta^{2}=c_{9} \eta^{2} .
$$

Denote

$$
L=\bigcup_{k=0}^{\infty} g^{-k}(K), \quad M=J_{g} \backslash L .
$$

LEMMA 13. If $x \notin L$ then $P(x)=\{x\}$.

Proof. Suppose that $P(x)$ is a proper interval. Then either $\tilde{g}^{k}(p(x))=\frac{1}{2}$ for some $k$, and then $x \in L$, or $g^{k}(P(x))$ is disjoint from $K$ for all $k \geq 0$. But in the second case (remember that we still assume that $K$ is a proper interval) it follows from [5] that the length of $g^{i}(P(x))$ grows exponentially with $i$ - a contradiction. In the only exception, when the periodic point of period $n$ in $K$ is semi-attracting and $g^{i}(P(x))$ comes arbitrarily close to $K$, we can use lemma 5 to get a contradiction.

LEMMA 14. Let $y \in L \cap J_{g}$ and $\varepsilon>0$. Then there exists $z \in J_{g}$ such that $g^{k}(z)=y$ for some $k \geq 0$ and the length of $P(z)$ is smaller than $\varepsilon$.

Proof. We consider three possible cases:

Case 1. $p(y) \notin\left\{\frac{1}{2}, \tilde{g}\left(\frac{1}{2}\right), \ldots, \tilde{g}^{n-1}\left(\frac{1}{2}\right)\right\}$. Then the set

$$
\bigcup_{k=0}^{\infty} \tilde{g}^{-k}(\{p(y)\}) \cap J_{\tilde{g}}
$$

is dense in $J_{\tilde{\mathbf{g}}}$ (and therefore infinite), and if $\tilde{g}^{k}(t)=p(y)$ then $\left.g^{k}\right|_{p^{-1}(\{t\})}$ is a homeomorphism onto $P(y)$. Hence, the conclusion of the lemma follows.

Case 2. $p(y) \in\left\{\tilde{g}^{3}\left(\frac{1}{2}\right), \ldots, \tilde{g}^{n-1}\left(\frac{1}{2}\right), \tilde{g}^{n}\left(\frac{1}{2}\right)\right\}$. Then $\left.g^{m-2}\right|_{P\left(1-g^{2}\left(\frac{1}{2}\right)\right)}$ is a homeomorphism onto $P\left(g^{m}\left(\frac{1}{2}\right)\right)(m=3,4, \ldots, n)$. Since

$$
1-g^{2}\left(\frac{1}{2}\right) \in J_{g}
$$

and

$$
p\left(1-g^{2}\left(\frac{1}{2}\right)\right) \notin\left\{\frac{1}{2}, \tilde{g}\left(\frac{1}{2}\right), \ldots, \tilde{g}^{n-1}\left(\frac{1}{2}\right)\right\}
$$

we can then use case 1 for $1-g^{2}\left(\frac{1}{2}\right)$.

Case 3. $p(y) \in\left\{\tilde{g}\left(\frac{1}{2}\right), \tilde{g}^{2}\left(\frac{1}{2}\right)\right\}$. Then $g$ maps $P\left(\frac{1}{2}\right)$ onto $P\left(g\left(\frac{1}{2}\right)\right) \cap J_{g}$ and $g^{2}$ maps $P\left(\frac{1}{2}\right)$ onto $P\left(g^{2}\left(\frac{1}{2}\right)\right) \cap J_{g}$. Since

$$
p\left(\frac{1}{2}\right)=\tilde{g}^{n}\left(\frac{1}{2}\right)
$$

we can then use case 2 . 
Now we go back to the general case and finish the proof of theorem B.

The first possibility is that $K$ consists of only one point, $\frac{1}{2}$. But since (by lemma 1)

$$
h(g) \geq \frac{1}{2} \log 2,
$$

this implies that $p$ is a conjugacy and $\left.\tilde{g}\right|_{J_{\tilde{\mathbf{s}}}}$ is transitive. Consequently, $\left.g\right|_{J_{\mathbf{g}}}$ is also transitive, and hence $\delta$-transitive for all $\delta>0$.

Thus, we remain again wih the case of $\frac{1}{2}$ periodic of period $n$ for $\tilde{g}$. We shall consider several possibilities for $x$ and $y$.

(a) $x, y \in M$. Since $\left.\tilde{g}\right|_{J_{\tilde{g}}}$ is transitive, there are $\tilde{g}$-orbits from an arbitrarily small neighbourhood of $p(x)$ to an arbitrarily small neighbourhood of $p(y)$. But, by lemma 13,

$$
P(x)=\{x\} \text { and } P(y)=\{y\} .
$$

Hence, if $p(z)$ tends to $p(x)$ (or $p(y)$ ) then $z$ has to tend to $x$ (or $y$ ). Therefore, there are $g$-orbits from an arbitrarily small neighbourhood of $x$ to an arbitrarily small neighbourhood of $y$.

(b) $x \in L$. Then there is $k$ such that $g^{k}(x) \in K$. By lemma 12 , there is a $g, c_{9} \eta^{2}$-pseudo-orbit $\left(x, g(x), \ldots, g^{k}(x), \tilde{x}\right)$ from $x$ to some $\tilde{x} \in M$.

(c) $y \in L \cap J_{\mathrm{g}}$. By lemma 14 , for any $\varepsilon>0$ we can find $\tilde{y} \in M$ and a $g$, $\varepsilon$-pseudoorbit $\left(\tilde{y}, z, g(z), \ldots, g^{k}(z)\right)$ from $\tilde{y}$ to $y$.

By combining (a), (b) and (c), we can get a $g, c_{9} \eta^{2}$-pseudo-orbit from $x$ to $y$ for any $x, y \in J_{\mathbf{g}}$. This ends the proof of theorem $\mathrm{B}$.

Remark. Since we need to use each of the operations described in (a), (b) and (c) at most once, we see that the sum of 'jumps' (i.e. distances between $g\left(x_{i}\right)$ and $x_{i+1}$ ) along our pseudo-orbit can be made smaller than $c_{9} \eta^{2}$.

\section{Example}

Let $g(x)=r x(1-x), \quad 0<r \leq 4$.

Then we can easily compute:

$$
\begin{gathered}
\eta=1-\frac{1}{4} r, \\
a^{\prime}=1-r^{-1}, \\
a=r^{-1}, \\
c_{1}=(r-2)(r-3) r^{-1}(r-1)^{-1}, \\
c_{2}=c_{3}=r, \\
c_{4}=2(r-2)(r-3) r^{-1}(r-1)^{-1}, \\
c_{5}=(r-1) r^{-1}, \\
c_{6}=\left[(r-1) r^{-1} \eta\right]^{\frac{1}{2}}, \\
c_{7}=3 r(r-1)(r-2)^{-1}(r-3)^{-1} \eta, \\
\frac{1}{16} c_{3}^{-2} c_{4} c_{5}^{-1} \ln ^{2}\left(c_{3} c_{5}+1\right)=\frac{1}{4}(r-2)^{2}(r-3)^{2} r^{-3}(r-1)^{-3} \ln ^{2} r, \\
\frac{1}{54} c_{3}^{-1} c_{4}^{2}=\frac{2}{27}(r-2)^{2}(r-3)^{2} r^{-3}(r-1)^{-2}, \\
a\left(g^{\prime}(0)\right)^{-1}=r^{-2},
\end{gathered}
$$




$$
\begin{gathered}
c_{8}=\min \left(\frac{1}{4}(r-2)^{2}(r-3)^{2} r^{-3}(r-1)^{-3} \ln ^{2} r,\right. \\
\left.\frac{2}{27}(r-2)^{2}(r-3)^{2} r^{-3}(r-1)^{-2}, r^{-2}, \frac{1}{2}\right), \\
c_{9}=9 r^{3}(r-1)^{2}(r-2)^{-2}(r-3)^{-2} .
\end{gathered}
$$

Since $\eta<\frac{1}{2}$, we have $1-\frac{1}{4} r<\frac{1}{2}$, and hence $r>2$. Then, since $\eta<a\left(g^{\prime}(0)\right)^{-1}$, we have $1-\frac{1}{4} r<r^{-2}<\frac{1}{4}$, and hence

$$
r>3 \text {. }
$$

From (3.13) and (3.15) it follows easily that

$$
c_{8}=\frac{2}{27}(r-2)^{2}(r-3)^{2} r^{-3}(r-1)^{-2} .
$$

Now we have:

$$
\begin{aligned}
\alpha & \leq 0.002 \Rightarrow 489 \alpha<1 \Rightarrow 486 \alpha<1-3 \alpha \\
& \Rightarrow \frac{\alpha}{4}<\frac{1-3 \alpha+2 \alpha^{2}}{4 \cdot 27 \cdot 18}=\frac{2 \cdot 4 \cdot(1-\alpha) \cdot(1-2 \alpha)}{2 \cdot 4 \cdot 4 \cdot 27 \cdot 2 \cdot 9} \\
& =\frac{2(4-4 \alpha)(1-2 \alpha)}{27 \cdot 4^{3} \cdot 3^{2}}<\frac{2(2-\alpha)^{2}(1-\alpha)^{2}}{27(4-\alpha)^{3}(3-\alpha)^{2}} .
\end{aligned}
$$

Hence, if $4-r \leq 0.002$, then

$$
\eta=1-\frac{r}{4}<\frac{2(r-2)^{2}(r-3)^{2}}{27 r^{3}(r-1)^{2}}=c_{8}
$$

Setting $\alpha=4 \eta$ again, we get

$$
\begin{aligned}
c_{9} & =\frac{9 r^{3}(r-1)^{2}}{(r-2)^{2}(r-3)^{2}}=\frac{9(4-\alpha)^{3}(3-\alpha)^{2}}{(2-\alpha)^{2}(1-\alpha)^{2}} \\
& <\frac{9 \cdot 4^{3} \cdot 3^{2}}{(4-4 \alpha)(1-2 \alpha)} \\
& =\frac{81 \cdot 16}{(1-\alpha)(1-2 \alpha)}<\frac{81 \cdot 16}{1-3 \alpha} .
\end{aligned}
$$

We have $\alpha=4 \eta$, and hence

$$
c_{9} \eta^{2}<\frac{81 \cdot 16}{1-3 \alpha} \frac{\alpha^{2}}{16}=\frac{81 \alpha^{2}}{1-3 \alpha} .
$$

If $\alpha \leq 0.002$ then

$$
\frac{81}{1-3 \alpha}<82
$$

Hence, we get the following result:

TheOREM C. If $0 \leq \alpha \leq 0.002$ then the map $x \mapsto(4-\alpha) x(1-x)$ is $82 \alpha^{2}$-transitive.

Part of this work was done while the author was at the University of Geneva and the author expresses his gratitude for their hospitality and support. 


\section{REFERENCES}

[1] L. Block, J. Guckenheimer, M. Misiurewicz \& L. S. Young. Periodic points and topological entropy of one-dimensional maps. Global Theory of Dynamical Systems, Lecture Notes in Math. No. 819, Springer: Berlin, 1980. pp. 18-34.

[2] P. Collet \& J.-P. Eckmann. Iterated maps on the interval as dynamical systems. Progr. in Phys. 1 Birkhäuser: Boston 1980.

[3] J. Guckenheimer. Sensitive dependence on initial conditions for one-dimensional maps. Commun. Math. Phys. 70 (1979), 133-160.

[4] J. Milnor \& P. Thurston. Kneading theory. Preprint, Princeton, 1977.

[5] M. Misiurewicz. Absolutely continuous measures for certain maps of an interval. Publ. Math. I.H.E.S. 53 (1981), 17-51. 